\title{
A Comparative Impact Study of Edible Oils on Health
}

\author{
Bharti ${ }^{*}$, Deepa Indoria, R.L. Solanki and B.S. Meena \\ Krishi Vigyan Kendra, Nr. Rithola toal Naaka, Chittorgarh-312001, India \\ *Corresponding author
}

\section{A B S T R A C T}

\begin{tabular}{|c|c|}
\hline Keywor & Dietary fats are closely related to Coronary Artery Disease (CAD). \\
\hline $\begin{array}{l}\text { Edible oil, Saturated } \\
\text { fats, Trans fats, } \\
\text { Omega } 3 \text { fats, N-6/N-3 } \\
\text { ratio, Smoking point, } \\
\text { mustard oil, Canola } \\
\text { oil. }\end{array}$ & $\begin{array}{l}\text { Cholesterol, saturated and trans fats are harmful whereas MUFA, PUFA } \\
\text { (especially Omega-3 PUFA) are beneficial for the heart. Choice of heart } \\
\text { healthy oil could decrease the occurrence of CAD. This article reviews the } \\
\text { benefits/harms of common edible oils on heart health. A heart healthy oil }\end{array}$ \\
\hline Article Info & \\
\hline $\begin{array}{l}\text { Accepted: } \\
\text { 07 September } 2017 \\
\text { Available Online: } \\
\text { 10 November } 2017\end{array}$ & $\begin{array}{l}\text { smoking points. Mustard and Canola oils offer to be ideal in this regard. } \\
\text { Epidemiologic studies also suggest that people consuming mustard oil have } \\
\text { less CAD as compared to other oils. }\end{array}$ \\
\hline
\end{tabular}

\section{Introduction}

Currently one is confronted by an array of cooking oils and a host of health claims about their respective benefits. To further confuse, several brands are available for each oil sporting all types of jargon on the package, refined/ filteredN-3, N-3 / N-6 ratio, enriched with oryzanol / omega 3 fatty acid, rich in Polyunsaturated (PUFA) / Monounsaturated (MUFA) fatty acids. It is the need of current time to clarify the health benefits of different oils and demystify the jargon for the lay people.

\section{Cholesterol}

It is the fatty substance that is found in the blood stream and the body cells. Actually, it is an essential substance required for a proper functioning of body especially the brain. Broadly, it is of two types: high density lipoprotein (HDL) and low density lipoprotein (LDL). LDL cholesterol is considered as "bad cholesterol" responsible for allowing fatty plaques to develop in the lumen of arteries, leading to their narrowing. If this narrowing develops in coronary arteries (supplying blood to the heart), the person can develop coronary artery disease (CAD) and can lead to heart attacks. HDL cholesterol on the other hand is called as "good cholesterol" and can prevent the development and evolution of these dangerous plaques.

It is important to remember that while fats of animal origin (butter, ghee, lard) may be rich in cholesterol, vegetable oils have no 
cholesterol, but some of them can lead to endogenous (by the body) production of cholesterol.

\section{Saturated fat (SFA)}

It is a type of fat in the oils which makes it thicker (more solid) and when consumed can elevate the levels of total cholesterol (TC) and LDL cholesterol. Overall, it can contribute to development of heart diseases. ${ }^{1}$

Rich sources: Ghee, butter and coconut oil

\section{Polyunsaturated fat (PUFA)}

It is another type of fat which when consumed lowers the level of LDL cholesterol but at the same time can also lower HDL cholesterol.

Rich sources: Safflower oil, sunflower oil and soya bean oil

\section{Monounsaturated fat (MUFA)}

Metabolically, it is the best type of fat because when consumed lowers LDL cholesterol and elevates the HDL cholesterol levels. $^{2-4}$

Rich sources: Olive oil, mustard oil and groundnut oil

However, currently, the focus of research has shifted from saturated fats to individual fats and percentage of fatty acids (SFA, PUFA, and MUFA) in the diet. An adequate intake of both polyunsaturated and saturated fats is needed for the ideal LDL/HDL ratio in blood, as both contribute to the regulatory balance in lipoprotein metabolism. ${ }^{5}$

\section{Trans fatty acids (TFAs)}

This is an artificial fat produced as a side effect of hydrogenation of animal fat (margarine) or vegetable fat (vanaspati ghee). If hydrogenation is incomplete (partial hardening), the relatively high temperatures used in the hydrogenation process tend to flip some of the carbon-carbon double bonds into the "trans" form. If these particular bonds aren't hydrogenated during the process, they will still be present in the final product as trans-fatty acid. From the health stand-point, it is the worst type of fat and has been linked with development of CAD. A comprehensive review of studies on trans fats was published in 2006 in the NEJM reports a strong and consistent relation between trans fat consumption and $\mathrm{CAD}$, concluding that "On a per-calorie basis, trans fats appear to increase the risk of CHD more than any other macronutrient, conferring a substantially increased risk at low levels of consumption (1 to $3 \%$ of total energy intake)". ${ }^{6}$ This study estimated that consumption of TFAs constituted for between 30,000 and 100,000 cardiac deaths per year in the United States alone. Another damaging evidence of illeffects of TFAs on CAD were forth-coming from the Nurses' Health Study, enrolling 120,000 female nurses. In this landmark study, $\mathrm{Hu}$ and colleagues determined that a nurse's CAD risk roughly doubled for only $2 \%$ increase in trans fat calories consumed.

Interestingly, it takes more than a $15 \%$ increase in saturated fat calories to produce a similar increase in risk. Furthermore, it was found that replacing $2 \%$ of food energy from TFAs with non-trans unsaturated fats more than halved the risk of CHD (53\%). By comparison, replacing a larger $(5 \%)$ of food energy from SFAs with non-trans unsaturated fats reduces the risk of $\mathrm{CHD}$ by $43 \%$. The reason for this exaggerated negative response is probably that while both SFA and TFA increase LDL but unlike SFA, TFA also lowers HDL. The net increase in LDL/HDL ratio with TFAs is approximately double than that due to SFAs. ${ }^{7}$ 
Furthermore, the negative consequences of TFA consumption may go beyond the CV risk. There is evidence that eating such diets may increase the risk of other chronic health problems like Alzheimer's disease, cancer, diabetes, obesity, liver dysfunction, infertility and depression to name a few.

Rich sources: Processed fried foods like bhujiya, samosas, biscuits, confectionary items, etc.

\section{Omega-3 fatty acids}

Omega-3(N-3) fatty acids are a family of PUFA, which are considered especially good for health. ${ }^{8}$ They are one of the two essential fatty acids, so called because humans cannot manufacture it and must get it from food. $\mathrm{N}$ 3fatty acids are precursors to antiinflammatory compounds in the body. Oily fish caught near polar regions are a good source of it. All seeds of the Brassica family, including mustard (6-11\%), canola / rapeseed (7\%) and turnip, have high levels of omega-3 fatty acid. Flax (linseed) oil is the richest source of plant based omega-3 fatty acid $(55 \%)$ and also hemp oil $(20 \%)$ is a rich source, but these oils are uncommon as a table or cooking oil (because of low smoke point). Soybean oil has $6 \%$ omega-3 but contains over $50 \%$ omega- 6 , the fatty acid that competes with the function of omega-3. Other than rapeseed and mustard oils, there are few other common sources of plant based omega3 in Western and Indian diets. However, when omega-6 intake is kept low, humans can convert the plant-based omega-3 into one found in fish oils (eicosapentaenoic acid), in limited amounts, a useful source for vegetarians.

\section{Omega-6 fatty acids}

Omega-6 fatty acids (N-6) are also important for health. They include the essential fatty acid linoleic acid (LA), which is abundant in vegetable oils like corn $(60 \%)$, cottonseed $(50 \%)$ and sunflower $(50 \%)$ oil. Margarine is very high in omega- 6 fatty acids. N-6 fatty acids are precursors to pro-inflammatory compounds in the body. Further, large amounts of omega- 6 decrease the effect of omega-3.

\section{N-6: $\mathrm{N}-3$ ratio}

Both N-6 and N-3 are essential for proper functioning of body, but they should be present in balanced proportion in the body. N6 and N-3 compete for the enzymes that convert them into more biologically active compounds. So, when more omega-6 is consumed, it uses proportionately more of those enzymes, ultimately leading to a more pro- inflammatory milieu. As per Institute of Medicine an adequate intake (AI) for N-6 is about 10 to 15 grams a day. The AI for N-3 is at least 1 to 2 grams a day. Although AIs are estimates and as always they vary depending on age, gender, life stage, activity level, so many things, it is recommended that the ratio in the diet should be less than4:1. On the other hand, World Health Organization (WHO) recommends $\mathrm{N}-6: \mathrm{N}-3$ ratio to be $5: 4$ and many consider In real world however, many people are eating a ratio of 20:1 and higher. N-6 and N-3 often occur together in foods. Many foods that are good sources of $\mathrm{N}-3$ are also good sources of N-6. Walnuts are a classic example in this case. They are rich in $\mathrm{N}-3$ but are even richer in N-6, so that the ratio is not $1: 1$ but closer to $4: 1$. Interestingly, with almonds the ratio is 1689:1. The only common foods where the ratio is reversed are the green leafy vegetables likeromaine, spinach, kale, broccoli, rapini, etc. Here, the $\mathrm{N}-6$ : $\mathrm{N}-3$ ratio is less than 1 and these are the foods which should be consumed more to improve the ratio. Among the cooking oils also it is very clear that those oils which are derived from green leafy vegetables, for 
example those derived from the Brasicca family like mustard oil or canola oil have the best N-6:N-3 ratio. Many popular oils like olive oil (12.84), corn oil (46.01), and peanut butter (225.63) have adverse ratios. On the other hand, mustard oil has a ratio of 1.2 which is closest to the one recommended by WHO i.e.

\section{Comparison of $\mathrm{N}-6$ / $\mathrm{N}-3$ ratios of common edible oil (Table 1)}

\section{Oryzanol}

Oryzanol is an antioxidant found in rice bran oil (RBO) that is used for many alternative herbal therapies. The high antioxidant property of $\square$-oryzanol has been widely recognized. Studies have shown that it can prevent heart attacks, probably because its effect on cholesterol metabolism; it can reduce plasma cholesterol, reduce cholesterol absorption and decrease early atherosclerosis, inhibit platelet aggregation, and increase fecal bile acid excretion. ${ }^{10}$ Oryzanol has also been used to treat nerve imbalance and disorders of menopause.

\section{Tocotrienols}

They belong to the vitamin E family and are powerful natural antioxidants and are abundantly found in palm oil, rice bran oil, coconut oil and also wheat germ and barley. Their protective benefit lies in the prevention of CVD and some forms of cancer.

In one study, it showed reversal of atherosclerosis in most patients of carotid atherosclerosis after consumption of tocotrienols (from palm oil) as compared to worsening in all the control patients. ${ }^{11}$ It acts perhaps by ability to decrease the liver's capacity to manufacture cholesterol by suppressing the HMG-CoA reductase (the enzyme in the liver responsible for cholesterol synthesis). ${ }^{12}$ Further, tocotrienols have also been found to have a neuro-protective (prevent stroke related injuries) and anticancer (prostatic, pancreatic, breast, and skin) effect. Some studies have also shown that palm tocotrienols improved blood glucose, dyslipidemia and oxidative stress and may prevent the progression of vascular wall changes occurring in Diabetes Mellitus (DM). ${ }^{13,14}$

\section{Phytosterols}

They are a class of naturally-occurring compounds found in plants that are analogous to cholesterol (function as a structural component to cell membranes). Vegetable and nut oils like wheat germ, sesame, corn, canola and almond oil are rich source of this oil.

Cholesterol lowering effects of phytosterols are well known. It has been reported that $2 \mathrm{~g}$ of phytosterols daily was associated with a $10 \%$ decrease in LDL. By virtue of lowering cholesterol it can even reduce the risk of CAD. In addition to supporting cardiovascular health, phytosterols may also reduce the risk of certain cancers. It may inhibit the growth and metastasis of cancers of the breast, ovary, lung and stomach probably by reducing oxidative stress associated with cancer. ${ }^{15}$

\section{Cold pressed oils}

This oil is produced by use of a hydraulic press. This is an ancient method and yields the best quality oil. The only two materials that will yield enough oil without heating them first are sesame seeds and olives. These oils are the closest possible to the natural state, therefore have the most color, odor and flavor - and are most nutritious; however they are usually unavailable because little oil is produced this way. 


\section{Refined oils}

Refined oil is purified oil obtained from oil cakes using a process of solvent extraction. Refined cooking oils are made by highly intensive mechanical and chemical (solvent extraction) processes to extract the oil from the seeds and vegetable products. The crushed seeds are heated to temperatures between $110^{\circ} \mathrm{C}$ and $180^{\circ} \mathrm{C}$ in a steam bath to start the oil extraction process. The seeds are put through a high volume press which uses high heat and friction to press the oil from the seed pulp. The seed pulp and oil are then put through a hexane solvent bath and steamed again to squeeze out more oil.

The extracted oil is then put through further refining techniques including degumming, neutralization and bleaching. Furthermore, high-temperature processing may cause the weak carbon bonds of unsaturated fatty acids, especially triple unsaturated linolenic acid, to break apart, thereby creating free radicals. In addition, antioxidants, such as fat-soluble vitamin $\mathrm{E}$, are neutralized or destroyed by high temperatures and pressures. Butylated Hydroxytoluene (BHT) and Butylated Hydroxyanisole (BHA), both suspected of causing cancer and brain damage, are often added to these oils to replace vitamin $\mathrm{E}$ and other natural preservatives destroyed by heat. It produces a bland oil having only mild flavor, free from impurities but more likely to turn rancid.

\section{Unrefined oils}

Unrefined oil is obtained by a process of pressing the seeds or other vegetable material. This uses a screw or continuous press with a constantly rotating worm shaft. Cooked material goes into one end and is put under continuous pressure until discharged at the other end with oil squeezed out. Temperatures between $200-250^{\circ} \mathrm{C}$ are normal. Obviously, this type of extraction does not qualify as "cold pressed" either. Organic merchants will refer to it as "expeller pressed". Health wise, unrefined oils are considered better and recommended due to presence of wide range of bioactive compounds (antioxidants), flavors and vitamin $\mathrm{E}$ content.

\section{Rancidity}

The term rancidity is used to designate the development of any disagreeable odor and flavor in fats and oils. Different investigators classify the disagreeable odors and flavors according to their production in different ways. Generally, there are three types of rancidities- (1) hydrolytic, (2) oxidative, and (3) ketonic.

\section{Hydrolytic rancidity}

Hydrolytic or the acid rancidity is brought about by the action of lipase enzymes which by hydrolysis split the fat into glycerol and fatty acids. Free fatty acids may also be liberated by a relatively high hydrogen-ion concentration in contact with the fat. Lipases are associated with fats in their natural state, i.e., nuts, seeds, milk, and fat of meat. Since lipase enzymes are destroyed by heat, this type of rancidity is encountered in products which are not heated to a high enough temperature to destroy the enzyme. The flavours developed by lipaseaction depend upon the composition of the fat. Thus, the flavors caused by butyric acid will be found only in products containing butter fat. As such lipase activity in itself is of no great economic importance, except in the fats rich in the lower fatty acids,but secondary reactions associated with oleicacidintroduce another aspect. The free fatty acids act ascatalysts for oxidative changes. The better-keeping quality of the sweet-cream butter is attributed to its lower free fattyacid content (compared to butter from sour cream) Oxidative rancidity 
Among the changes which occur when a fat or oil becomes rancid are the following: the iodine value decreases, whereas the specific gravity, acid value, and peroxide value increases. Investigations have shown that when an oil or fatis protected from light by means of a green wrapper or container it may have a peroxide value equal to or even greater than an unprotected fat that has become rancid. Oxidative rancidity occurs through the taking up of oxygenat the double bonds of the unsaturated glycerides. Many oxidative decomposition products may be formed. These products include aldehydes, ketones, fatty acids of lower molecular weight, hydroxy acids, oxy acids, and gases. The gaseous decomposition products of rancid fats are carbon dioxide, carbon monoxide, hydrogen, nitrogen, oxygen, and other gases.

\section{Induction period}

There is a period before the uptake of oxygen by a fat becomes appreciable which is known as the induction period. During this period the fat is still fresh and "sweet." The induction period varies for different fats and oils and for different samples of the same fat or oil. The oxidation products act as catalysts so that oxygen uptake receives increased momentum as these products are formed. The first compounds formed in oxidation of unsaturated fatty acids are "moloxides." (not oxides or peroxides). Exclusion of air or oxygen from a fat may retard but not inhibit its oxidation. Any particular oil can be prevented from getting rancid bydelaying the development of rancidity, a blanket of an inert gas, usually nitrogen, is applied to the vapor space in the storage container immediately after production. This is referred to as tank blanketing. Vitamin $\mathrm{E}$ oil is a natural antioxidant that can also be added to cooking oils to prevent rancidification. All oils should be kept in a cool, dry place. Oils may thicken, they will soon return to liquid if they stand at room temperature. To prevent negative effects of heat and light, oils should be removed from cold storage just long enough for use. Refined oils high in MUFA keep up to a year (olive oil will keep up to a few years), while those high in PUFA keep up to about six months. In contrast, saturated oils, such as coconut oil and palm oil, have much longer shelf lives and can be safely stored at room temperature. Their lack of PUFA content causes them to be more stable.

\section{Smoking temperatures of fats (Smoke point/ flash point)}

When fats or oils are heated to high temperature, decomposition occurs and finally a point is reached where fat is broken down to glycerol and free fatty acids (FFA), and produce bluish smoke (visible fumes are given off). This point is called the "smoke point." The glycerol is then further broken down to acrolein which is also a component of the smoke. It is the presence of the acrolein that causes the smoke to be extremely irritating to the eyes and throat. The smoke point also marks the beginning of both flavor and nutritional degradation giving an unpleasant and disagreeable flavor to the food. Therefore, it is a key consideration when selecting a fat for frying (requiring fats with higher smoke points for deep frying).

The smoke point for oil varies widely depending on origin and refinement. The smoke point of oil does tend to increase as FFA content decreases and degree of refinement increases. ${ }^{16,17}$ Lighter, more refined oils tend to have higher smoke points. Reused oil has a lower smoke point because heating the oil produces FFAs and as heating time increases, more such acids are produced, thereby decreasing the smoke point.

Considerably above the temperature of the smoke point is the "flash point", the point at 
which the vapors from the oil can first ignite when mixed with air. Even if smoking point is not reached even high heating can cause harm with some oils. A 2001 parallel review of 20- year dietary fat studies in the UK, the USA, and Spain found that PUFA containing oils like soya, canola, sunflower, and corn oil degrade easily to toxic compounds

Table.1 Comparison of fats available in different oils

\begin{tabular}{lccccc}
\hline Fats/Oil & $\begin{array}{c}\text { Saturated } \\
\text { Fatty Acid (SFA) }\end{array}$ & $\begin{array}{c}\text { Mono-unsaturated } \\
\text { Fatty Acid (MUFA) }\end{array}$ & $\begin{array}{c}\text { Linoleic Acid } \\
\text { (Omega-6 PUFA) }\end{array}$ & $\begin{array}{c}\text { Alpha-Linoleic Acid N-6/N-3 ratio } \\
\text { (Omega-3 PUFA) }\end{array}$ \\
Mustard & 8 & 70 & 12 & 10 & $1.2: 1$ \\
Canola & 4 & 62 & 22 & 10 & $2.2: 1$ \\
Ghee & 65 & 32 & 2 & $<1$ & $3: 1$ \\
Soybean & 15 & 27 & 53 & 5 & $10.6: 1$ \\
Red palm & 50 & 40 & 9 & $<0.5$ & $18: 1$ \\
Olive oil & 13 & 76 & 10 & $<0.5$ & $20: 1$ \\
Palm oil & 45 & 44 & 10 & $<0.5$ & $20: 1$ \\
Rice bran & 22 & 41 & 35 & 1.5 & $23: 1$ \\
Groundnut & 24 & 50 & 25 & $<0.5$ & $50: 1$ \\
Sunflower & 13 & 27 & 60 & $<0.5$ & $120: 1$ \\
Safflower & 13 & 17 & 70 & $<0.5$ & $140: 1$
\end{tabular}

Source: POS Pilot Plant Corporation, Saskatoon, Saskatchewan, Canada, June 1994; National Institute of Nutrition, India

\section{Which oils to use?}

Several oils are available but oils should be chosen on the intended use (oils with high smoke point for deep frying), ability to stay on shelf and also the fat composition, more importantly the ratio between PUFA, MUFA, SFA (N-3, N-fat components (Table 1). However, many oils have some special component or characteristic which makes them especially useful.

\section{Mustard oil}

This is oil used primarily in North India and East India. It has a hot nutty taste and pungent aroma. Mustard oil has about $70 \%$ MUFA of which $42 \%$ is erucic acid and $12 \%$ is oleic acid, it has $22 \%$ PUFA content of which 10 $\%$ is the omega-3 alpha-linolenic acid and 12 $\%$ omega-6 linoleic acid and $8 \%$ SFAs. Mustard oil also have high levels of omega-3 (10 \%) and are a common, cheap, massproduced vegetarian source of omega-3 fatty acids. It was once considered unsuitable for human consumption due to high content of erucic acid. This is because of early studies in rats. However, later studies showed that in rats there is an inefficient activation of erucic acid to erucyl-CoA and a low level of activity of triglyceride lipase and enzymes of betaoxidation for erucic acid which possibly contribute to the accumulation and retention of cardiac lipid.

Mustard oil is considered one of the healthiest edible oils as it has low amount of SFAs and a high amount of MUFA and PUFA fatty acids, which are good for health. Compared to other oils mustard oil has several benefits. Furthermore, the N-6: N-3 ratio of mustard oil is near ideal 6: 5 unlike olive oil where it is 20:1. It has a high content of antioxidants and vitamin $E$, as well as the fact that it is cold pressed add to the nutrition value of this oil. The slphalinolenic acid found in mustard oil reduces the adhesion-aggregation tendency of blood platelets which decreases the risk of a 
heart attack. Several, in human studies (on postmortem samples) early asymptomatic coronary disease was found absent in the mustard suggesting that mustard oil may be cardio- protective. Several clinical studies have also found that mustard oil may be the best for heart health.

A study by Harvard school of medicine, All India Institute of Medical Sciences (AIIMS), Delhi and St. John Hospital, Bangalore, found that there was a significant and dosedependent inverse association between vegetable intake and Ischemic heart disease (IHD) risk. The inverse association was stronger for green leafy vegetables. Use of mustard oil, which is rich in linolenic acid, was associated with a lower risk than was use of sunflower oil [for use in cooking: RR: 0.49 (95\% CI: 0.24, 0.99); for use in frying, RR: 0.29 (95\% CI: 0.13, 0.64)]. In fact the chances of heart disease drop by nearly $70 \%$ on use of mustard oil as a cooking medium. ${ }^{19,20}$

\section{Rapeseed oil (Canola oil)}

It is plant-based oil, a close relative of mustard (Brassica family). Recent varieties have lower levels of erucic acid. The name "Canola" was derived from the term "Canadian oil, low acid" in 1978.21Canola oil is low in SFAs (less than 7\%), is high in MUFA, and has a beneficial omega-3 fatty acid profile (which has well established heart health benefits) and is recognized by many health professional organizations including the American Dietetic Association (ADA), and American Heart Association (AHA). Canola oil has been considered healthy food by FDA as well. ${ }^{22}$

\section{Olive oil}

Olive oil is oil obtained from the olive, a traditional tree crop of the Mediterranean basin. Olive oil is considered the best oil by some because it has the highest (75 \%) content of MUFA among all other oils. Furthermore, it also contains a wide variety of valuable antioxidants (hydroxytyrol is the main antioxidant compound in olive oil), believed to play a significant role in the many health benefits attributed to olive oil. The use of MUFA (most notably oleic acid) leads lowering of LDL cholesterol and elevation of HDL cholesterol which may lead to reduction in the risk of CAD. ${ }^{23}$ Furthermore; it exerts anti-inflammatory, antithrombotic, antihypertensive as well as vasodilatory effect. It must be noted that the least processed forms of olive oil, extra virgin or virgin olive oil have more MUFA than other olive oil. In addition, they also contain more polyphenols, which may be responsible for beneficial effects of this oil on heart. ${ }^{24}$

The polyphenolic antioxidants (oleuropein or tyrosol) which are found in highest concentration in extra-virgin olive oil have been co-related with increased arterial elasticity which itself leads to decreased risk of stroke and heart attacks.

Not only that some epidemiological studies suggest that olive oil has a protective effect against certain malignant tumors in the breast, prostate, endometrium and digestive tract. ${ }^{25}$ The main limitation of this oil is that it does not have an ideal N-6: N-3 ratio.

\section{Soyabean oil}

It is one of the most widely consumed cooking oils. Like safflower and sunflower oil, it is also a rich source of PUFA (although less than them).

The major unsaturated fatty acids in soybean oil triglycerides are 7-10\% alpha-linolenic acid (C-18:3); 51\% linoleic acid (C-18:2); and $23 \%$ oleic acid (C-18:1). It also contains the saturated fatty acids $4 \%$ stearic acid and 
$10 \%$ palmitic acid which are long chain saturated fatty acids. Like safflower oil and sunflower oil it has a high content of linolenic acid and as such tends to turn rancid earlier and therefore should be used fresh.

It is generally recommended that to achieve best health benefits, oils rich in PUFA should be used in combination with those rich in MUFA like olive oil, mustard oil or groundnut oil.

\section{Rice bran oil (RBO)}

RBO is oil extracted from the germ and inner husk of rice. It is notable for its very high smoke point of $254 \mathrm{C}$ (thus utilized for deep frying) and its mild flavor. It is one of the most popular cooking oils in Japan and China. Rice bran oil is perhaps one of the most balanced oils containing a range of fats $(47 \%$ MUFA, 33\% PUFA, and 20\% SFA. It is also rich in vitamin $\mathrm{E}$, gamma-oryzanol (a powerful antioxidant) which may help prevent heart attacks), and phytosterols (compounds believed to help lower cholesterol absorption), which may provide associated health $h$ benefits. A major limitation of this oil is less than ideal N-6/N-3 ratio of 23:1.

\section{Sunflower oil}

It is like safflower oil which is high in PUFA and the essential vitamin $\mathrm{E}$ and low in SFA. In addition it is rich in lecithin, tocopherols, carotenoids and waxes. Practically, it is good cooking oil known for having a clean taste and low levels of trans- fat. However, it also has a high content of omega-6 polyunsaturated fatty acids. Health related data associated with high consumption of mega 6 polyunsaturated fatty acid is controversial. Some studies have suggested that it may increase the likelihood of breast and prostatic cancer. Another major limitation of this oil

\section{Cotton seed oil}

It is cooking oil extracted from the seeds of cotton plant of various species. Its fatty acid profile generally consists of $18 \%$ MUFA (oleic), and 52\% PUFA(linoleic \& linolenic). Cottonseed oil is described by scientists as being "naturally hydrogenated" because the SFAit contain sare the natural myristic, palmitic, and (predominantly) stearic acids. These fatty acids make it stable frying oil without the need for additional processing or the formation of trans- fatty acids. However, the health benefits of this oil are controversial, it has too high acontent of saturated fat and too low in monounsaturated fat. ${ }^{29}$ Furthermore, cottonseed oil may contain natural toxins and unacceptably high levels of pesticide residues (since "cotton is not classified as a food crop, and farmers use many agrichemicals when growing it).

The natural toxin, gossypol, is supposedly eliminated in the refining process. The main advantage of cottonseed oil is that it is naturally rancid free (and may not require hydrogenation to make it stable) and therefore has a long shelf life. Being cheap it is now a component of a much wider range of processed foods, including cereals, breads and snack foods like chips.

\section{Corn oil}

Corn oil (Maize oil) is oil extracted from the germ of corn. Refined corn oil is approximately 55\% PUFA, 30\% MUFA, and $15 \%$ SFA. Its main use is in cooking, where its high smoke point makes it valuable for frying purposes.

It is also relatively inexpensive. However, refined corn oil has excessive levels of omega- 6 fatty acids, relative to omega- 3 fatty acids, may increase the probability of a number of diseases and depression. 


\section{Ground nut oil}

It is oil most often used in Chinese, South Asian and Southeast Asian cuisine. Peanut oil is appreciated for its high smoke point relative to many other cooking oils. In addition, it has a pleasing taste that can add additional character to a dish. Its major component fatty acids are oleic acid (MUFA), linoleic acid (PUFA), and palmitic acid (SFA). The oil also contains some stearic acid, arachidic acid, arachidonic acid, behenic acid, lignoceric acid and other fatty acids. This is one of the heart friendly oils which are not only rich in MUFA but otherwise also well balanced.

\section{Safflower oil}

Safflower produces two types of oil but the common one available is Indian market is high in PUFA (linoleic acid). Being low in SFA, it was initially used as a substitute for ghee and butter as health consciousness developed in India. However, being rich in PUFA and very low in MUFA (13\%), it is also not the healthiest of oils. While its consumption may cause the lowering of total and LDL cholesterol it may also cause lowering of HDL cholesterol. Being high in PUFA it is more likely to turn rancid and although it has the highest smoke point and as such can be used for deep frying, it must be remembered that poly-unsaturated fat possibly turn toxic when exposed to high heat.

\section{Palm oil}

Palm oil is extracted from the pulp of the fruit of the oil palm. Palm oil is a common cooking ingredient in the tropical belt of Africa, Southeast Asia and parts of Brazil. It is cheap and has a high smoke point and thus useful for frying. One variety, the red palm oil contains at least 10 other carotenes, along with tocopherols and particularly, tocotrienols, Coenzyme Q10, phytosterols and glycolipids. Though palm oil may be high in saturated fat, the co-relation between heart disease and this oil is controversial. While a study (supported by the NIH and the USDA) concluded that palm oil is not a safe substitute for trans-fat in the food industry, because palm oil results in adverse changes in the blood concentrations of LDL cholesterol and apolipoprotein B similar to trans fat does. ${ }^{30}$ Recent researches have shown that saturated fats in the sn- 1 and -3 positions of triacylglycerol exhibit different metabolic patterns due to their low absorptivity. Thus, dietary fats containing saturated fats primarily in sn-1 and -3 positions (e.g., cocoa butter, coconut oil, and palm oil) have very different biological consequences than those fats in which the saturated fats are primarily in the sn-2 position (e.g., milk fat and lard). More studies should be carried out on effect of differences in stereospecific fatty acid location and in health outcomes. Major limitations of this oil are high saturated fat content and less than ideal N-6: N-3 ratio of 20:1.

\section{Coconut oil}

Throughout the tropical world it has provided the primary source of fat in the diets of millions of people for generations. Being a plant fat, it contains no cholesterol but consists of more than ninety percent of SFAs, with traces of few unsaturated fatty acids, such as MUFA and PUFA.

However, the saturated fat content is different from animal fats because most of them are medium chain triglycerides (which may not carry the same risks as other saturated fats). ${ }^{30}$ Lauric Acid is the chief contributor, with more than $40 \%$ of the share, followed by capric acid, caprylic acid, myristic acid and palmitic. Lauric acid is a saturated fat that raises blood cholesterol levels by increasing 
the amount of HDL cholesterol. It also contains vitamin $\mathrm{E}$ and vitamin $\mathrm{K}$ and minerals such as iron.

\section{Butter}

It is a fat derived from animal source and is high in SFAs and cholesterol as such it can raise total and LDL cholesterol (but may also elevate HDL cholesterol). It is a good flavoring agent and can be used for baking and preparing sauces but having a low smoke point; therefore cannot be used for deep frying. Ghee It is basically clarified butter but it can be used even for frying because it has got a higher smoke point.

\section{Vanaspati ghee}

It is essentially a refined vegetable oil which has been made more stable. As such it has much more shelf life and is least likely to turn rancid. However, it is not ideal for deep frying as it has a relatively low smoke point.

Furthermore, it is the unhealthiest of all oils because not only is it devoid of natural vitamins and bio-active compounds but as a result of hydrogenation process it contains an undesirable type of fat called trans fat. Consumption of this fat not only leads to the worst type of lipid profile possible but also has been directly co-related with development of CAD.

Review of various edible oils has been presented and their benefits and harmful effects on heart health have been discussed. Out of several edible oils available, mustard and rapeseed oil appear to be most heart healthy as they are low in saturated fat, high in MUFA and PUFA and they have the highest N-3/N-6 ratio. Epidemiological studies also suggest that mustard oil can decrease the occurrence of coronary artery disease

\section{References}

Ascherio A, et al., Trans fatty acids and coronary heart disease. $\mathrm{N}$ Engl J Med. 1999; 340(25): 1994-8.

Berquin IM, et al., Modulation of prostate cancer genetic risk by omega-3 and omega-6fattyacids. J Clin Invest. 2007; 117(7): 1866-75.

Bester D, Esterhuyse, Truster EJ, van Rooyen J. Cardiovascular effects of edible oils: a comparison between four popular edible oils. Nutr. Res. Rev. 2010; 23: 334-48

Bockisch M. Fats and Oils Handbook. Champaign, IL: AOCS Press. 1998 pp. 95-6.

Brown J, Thill DC, Brown AP, Mallory-Smith C, Brammer TA and Nair HS. Gene transfer between canola (Brassica napus L.) and related weed species. Annals of Applied Biology 1996; 129 (3): 513-22.

Budin SB, et al., The effects of palm oil tocotrienol-rich fraction supplementation on biochemical parameters, oxidative stress and the vascular wall of streptozotocin-induced diabetic rats. Clinics (Sao Paulo). 2009; 64(3):235-44.

Charlton KM, et al., Cardiac lesions in rats fed rapeseed oils. Can. J. Comp. Med. 1975; 39 (3): 261-9.

Cicero AF, Gaddi A. Rice bran oil and gammaoryzanol in the treatment of hyper lipoproteinaemias and other conditions. Phytother Res 2001; 15 (4): 277-286.

Cicero AF, Gaddi A. Rice bran oil and gammaoryzanol in the treatment of hyper lipoproteinemias and other conditions. Physiother Res, 2001; 15: 277-86.

De Lorgeril M, Salen P. The Mediterraneanstyle diet for the prevention of cardiovascular diseases. Public Health Nutr. 2006; 9(1A):118-23.

Demonty I, et al., Continuous dose-response relationship of the LDL-cholesterollowering effect of phytosterolin take. J Nutr. 2009; 139(2):271-84.

Hayes, Kenneth, Khosla, Pramod. The complex interplay of palm oil fatty acids on blood 
lipids. European Journal of Lipid Science and Technology 2007; 109 (4): 453.

$\mathrm{Hu}$ FB, Manson JE, Willett WC. Types of dietary fat and risk of coronary heart disease: A critical review. J Am Coll Nutr. 2001; 20(1):5-19.

Hu FB, Stampfer MJ, Manson JE, et al., Dietary fat intake and the risk of coronary heart disease in women. N Engl J Med. 1997; 337(21):1491-9.

Jeppesen et al., Low triglycerides-high -density lipoprotein cholesterol and risk of ischemic heart disease. J Am Coll Nutr; 20(1):5-19.

Keys A, et al., The diet and 15-year death rate in the seven countries study. Am. J. Epidemiol. 1986; 124 (6): 903-15.

Parker RA, et al., Tocotrienols regulates cholesterol production in mammalian cells by post-transcriptional suppression of 3-hydroxy-3-methylglutaryl-coenzyme Areductase. J Biol Chem. 1993; 268(15):11230-8.

Kuhad A, et al., Suppression of NF-kappabeta signaling pathway by tocotrienol can prevent diabetes associated cognitive deficits. Pharmacol Biochem Behav. 2009; 92(2):251-9.

Martin G, et al., Health effects of oxidized heated oils. Foodservice Research International 2001; 13: 41-55.

Morgan DA. Smoke, fire, and flash points of cottonseed, peanut, and other vegetable oils. Journal of the American Oil Chemists' Society 1942; 19(11): 193-198,

Mozaffarian D, et al., Trans fatty acids and cardiovascular disease. $\mathrm{N}$ Engl J Med. 2006; 354(15):1601-13.
Muller, et al., The Serum LDL/HDL cholesterol ratio is influenced more favorably by exchanging Saturated with unsaturated fat than by reducing saturated fat in the diet of women. J.Nutr. 2003; 133(1): 78-83.

Rastogi T, et al., Diet and risk of ischemic heart disease in India. Am. J. Clin. Nutr. 2004; 79 (4): 582-92.

Rastogi T, et al., Diet and risk of ischemic heart disease in India. Am. J. Clin. Nutr. 2004; 79 (4): 582-92.

Sonestedt E, Ericson U, Gullberg B, Skog K, Olsson H, Wirfalt E. Do both heterocyclic amines and omega -6 polyunsaturated fatty acids contribute to incidence of breast cancer in post-menopausal women of Malmo diet and cancer cohort? The International Journal of Cancer 2008; 123 (7): 1637-1643.

Tarrago-Trani MT, et al., New and existing oils and fats used in products with reduced trans-fatty acid content. J Am Diet Assoc. 2006; 106(6):867-8

Tomeo AC, et al., Antioxidant effects of tocotrienols in patients with hyperlipidemia and carotidstenosis. Lipids. 1995; 30(12):1179-8 J. Preventive Cardiology Vol. 1 No. 3 February 2001

Turner R, et al., Antioxidant and antiatherogenic activities of olive oil phenolics. Int J VitamNutr Res. 2005; 75 (1): 61-70.

Vega-Lopez S, et al., Palm and partially hydrogenated soybean oils adversely alter lipoprotein profiles compared with soybean and canola oils in moderately hyperlipidemic subjects. Am J Clin. Nutr. 2006; 84(1):54-62.

\section{How to cite this article:}

Bharti, Deepa Indoria, R.L. Solanki and Meena, B.S. 2017. A Comparative Impact Study of Edible Oils on Health. Int.J.Curr.Microbiol.App.Sci. 6(11): 601-612. doi: https://doi.org/10.20546/ijcmas.2017.611.072 